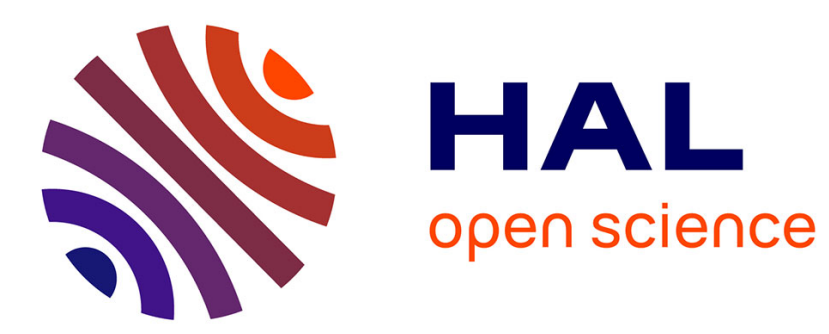

\title{
The Role of Losses in the Definition of the Overmoded Condition for Reverberation Chambers and Their Statistics
}

\author{
Andrea Cozza
}

\section{- To cite this version:}

Andrea Cozza. The Role of Losses in the Definition of the Overmoded Condition for Reverberation Chambers and Their Statistics. IEEE Transactions on Electromagnetic Compatibility, 2011, 53 (2), pp.296-307. 10.1109/TEMC.2010.2081993 . hal-00530840

\section{HAL Id: hal-00530840 https://hal.science/hal-00530840}

Submitted on 30 Oct 2010

HAL is a multi-disciplinary open access archive for the deposit and dissemination of scientific research documents, whether they are published or not. The documents may come from teaching and research institutions in France or abroad, or from public or private research centers.
L'archive ouverte pluridisciplinaire HAL, est destinée au dépôt et à la diffusion de documents scientifiques de niveau recherche, publiés ou non, émanant des établissements d'enseignement et de recherche français ou étrangers, des laboratoires publics ou privés. 


\title{
The Role of Losses in the Definition of the Overmoded Condition for Reverberation Chambers and Their Statistics
}

\author{
Andrea Cozza \\ Département de Recherche en Électromagnétisme, SUPELEC \\ 3 rue Joliot-Curie, 91192 Gif-sur-Yvette, France
}

\begin{abstract}
It is commonly acknowledged that in perfectlystirred reverberation chambers the energy density of the electric field follows a $\chi_{6}^{2}$ law, as long as the overmoded condition applies. This concept, never defined properly, is often confused with the idea of a threshold on the modal density, regardless of the quality factor of the cavity. This interpretation is here proven to be inaccurate, as losses play a fundamental role in the nature of the field statistics and not, as often assumed, just in its scaling. In particular, it is shown how the overmoded condition should be stated mathematically, highlighting how the cavity quality factor and the number of eigenmodes excited cannot be regarded as quantities intervening independently on the field statistics, but should rather be considered jointly. These results are derived by means of a modal analysis, with a limited number of assumptions. A quantitative relationship is established between average modal overlapping and the rate of convergence of the electric energy density towards a $\chi_{6}^{2}$ law. Rather than setting an arbitrary threshold on modal overlapping as a necessary condition for an overmoded behaviour, the statistical uncertainty due to the limited number of available field samples is shown to affect the very definition of the overmoded condition. Numerical as well as experimental results support our conclusions.
\end{abstract}

\section{INTRODUCTION}

Current use of reverberation chambers is based on a number of commonly accepted rules. Among these, the fact that an overmoded condition is necessary to achieve isotropy, uniformity and depolarization of the electromagnetic field in a test volume can be regarded as one of the most fundamental [1], [2], [3]. It is hence surprising that the study of this condition has not received much attention: to the best of our knowledge, no clear definition has yet been given in the field of Electromagnetic Compatibility, even though a similar criterium exists in acoustical reverberation chambers [4], albeit unable to provide an assessment of the rate of convergence of field statistics to theoretically justified asymptotic laws

As a matter of fact, going through the literature, it appears that the concept of an overmoded cavity is somewhat regarded as related to a threshold value in the modal density [2]. This likely comes from the fact that the availability of a large number of modes resonating at the working frequency is necessary, if the field distribution inside the cavity is to be complex enough to behave as a random distribution under the use of a stirring technique, e.g., by rotating an electrically large mechanical paddle [1], [3]. To the best of our knowledge, no study has yet clearly defined this threshold level, although the strong ties between a high modal density and a well-stirred cavity are known in practice [1]. A significant example of the low interest the overmoded condition aroused in most previous works is given in [1], where the overmoded condition is dismissed as something seemingly trivial, fulfilled as soon as a cavity is electrically large. Interestingly, Lehman [3] regarded the assessment of the validity of the overmoded condition as an open issue.

Fulfilling the requirement for an overmoded cavity allows the use of simplified models, as the one presented in [5], based on the description of the electromagnetic field as a continuous plane-wave spectrum: it links in a straightforward manner the idea of a well-stirred cavity to field statistics: an environment where an infinite number of plane waves propagate with the same probability along all the directions and polarizations, implies that the electric energy density follows a $\chi_{6}^{2}$ law, as a direct consequence of the central-limit theorem [6], [3]. Since this theoretical result is based on the assumption of a well-stirred cavity, the limited efficiency of the stirring technique is often regarded as the most likely source of noncompliancy, especially in the lower frequency range, because of the statistical correlation of contiguous stirrer positions [7].

But another potential reason of non-compliancy could come from the inadequacy of the assumption of an infinite number of propagating plane waves (intrinsically linked to the use of a continuous plane-wave spectrum) as this condition is approximatively fulfilled only asymptotically. In practice, depending on the number of resonant modes excited at the working frequency, the number of plane waves into which the field can be decomposed is finite, hence resulting in a nonperfect matching between a $\chi_{6}^{2}$ probability law and what is observed from experimental data about electric energy density. Users of reverberation chambers widely consider that working at frequencies above the Lowest Usable Frequency (LUF), as defined in [8], is a sufficient condition to make a cavity overmoded. This idea has already been proven to be incorrect [9], [10], as the statistical properties of the field generated at frequencies close to the LUF can be quite different from the ideal asymptotic case treated in most statistical models of reverberation chambers. Nevertheless, it is well accepted that working at frequencies well above the LUF ensures an overmoded condition.

All these results point to the fact that the occurrence of a non-compliancy at high frequency is unlikely, as both the modal density and the stirrer efficiency are expected to be high. 
These facts seem to support again the idea that the overmoded condition is linked to a threshold in the modal density, without taking into account the role of losses. Losses are merely included a posteriori, when computing the efficiency of a cavity in converting an input power into a high-intensity field. An exception is the analysis of how increasingly high losses impact statistical uniformity, presented in [11], where the requirement for a minimum quality factor was investigated. In this paper, we deal with configurations that are on the other end of the scale: as a matter of fact, the reverberation chamber will always be assumed to be highly resonant, with a quality factor much higher than one, typically several hundredths. Additional losses will always be assumed only to affect the relative bandwidth of resonant modes, with no influence on the way resonances are established.

Under these conditions, experimental data have been presented in [12], [13], [14], [15], providing clear clues that the overmoded condition is actually not based on a threshold level for the modal density and that losses can have a beneficial impact on field statistics [12], [13]. A similar conclusion was also suggested in a study based on a canonical modal representation [16], though the lack of an analytic approach hindered the development of predictive/design tools; moreover, the possibility of non-compliancy above the LUF was not pointed out. Indeed in [14], [15], no doubt could subsist about the stirrer efficiency at those frequencies where the field statistics was shown not to comply with the asymptotic probability laws predicted by continuous plane-wave spectrum models, especially because they occurred over a small subset of scattered frequencies.

This gives room to the idea that the overmoded condition is not just a matter of having a large number of modes and an ideally perfect stirrer. An eventual role of losses in field statistics would also cast some doubts on the often invoked idea of unstirred components. As soon as field statistics do not comply with asymptotic ones, this is regarded as due to a bad stirring. The results shown in this paper prove that statistical non-compliance can also be explained by a weak modal overlapping, even though a perfect stirring is assumed and a large number of resonant modes are potentially available.

This paper proposes a theoretical analysis linking in a formal way the statistical properties of the electromagnetic field within a reverberation chamber and two of its most important quantities: the composite quality factor and the number of modes excited at the working frequency. It will be shown that losses must be included into modal representations, in order to derive a meaningful statistical analysis of the field within a reverberation chamber. A modal approach is employed to this effect, staging a finite number of resonant modes excited at a given frequency. We will not address the question of statistical uniformity, but rather that of statistical convergence for the electric energy density measured at a given position.

The paper is organized as follows: Section II recalls the basics of modal analysis as derived for metallic enclosures; some important notations and concepts are introduced for the benefit of the derivations presented in the rest of the paper. Section III makes use of this modal representation, applying the concept of statistical excitation of the chamber, in order to derive the standardized variance of the electric energy density. Following these results, it is shown how the composite quality factor and the modal density of the chamber impact field statistics, and in particular the fact that the theoretical asymptotic behaviour predicted in [5] can be disproved. This leads to a quantitative definition of the overmoded condition in Section IV, as the one ensuring a limited error with respect to asymptotic statistics. Section V then seeks to assess how this deviation affects the probability of rejection in goodness-of-fit tests on field samples. This is achieved by means of numerical simulations based on the proposed modal representation. Experimental results are then presented in Section VI to check the validity of these ideas.

\section{MODAL REPRESENTATION AND NOTATIONS}

As we are interested in the statistical properties of the field excited within a cavity, we will make use of a modal representation, being an effective tool to this effect. We shall thus express the electric field as follows [17], [18]

$$
\mathbf{E}(\mathbf{r}, f)=\sum_{i=1}^{\infty} \gamma_{i}(f) \mathbf{e}_{i}(\mathbf{r}, f) \psi_{i}(f),
$$

where $\mathbf{r}$ is the position at which the field is being observed and $f$ the working frequency for a harmonic excitation. Three sets of modal quantities are involved in (1): 1) the modal weights $\left\{\gamma_{i}(f)\right\}$, which depend only on frequency for a given configuration of the excitation sources; 2) the modal topographies $\left\{\mathbf{e}_{i}(\mathbf{r}, f)\right\}$ describing the spatial dependence of the field for each mode and 3$)$ the frequency responses $\left\{\psi_{i}(f)\right\}$ of the resonant modes. The computation of the modal weights requires a precise knowledge of the modal topographies $\left\{\mathbf{e}_{i}(\mathbf{r}, f)\right\}$ as the former are obtained by projecting the equivalent current distribution of the sources over the modal topographies [17].

As rightfully recalled in [2], though this approach is exact, in practice it is hardly usable, as the computation of the modal topographies comes, apart for canonical configurations, as a computational burden for most numerical codes. This notwithstanding, the modal approach allows deriving some fundamental results, as will be proven here. To this end, a number of simplifications are required, enforced on three sets of modal parameters.

Let us recall that the $\left\{\psi_{i}(f)\right\}$ represent the responses of second-order systems, defined as follows

$$
\psi_{i}(f)=\frac{f}{f_{i}^{2}\left(1+\mathrm{j} / 2 Q_{i}\right)^{2}-f^{2}},
$$

where $f_{i}$ is the resonance frequency of the $i$-th mode and $Q_{i}$ its quality factor. The first set of modal parameters is thus given by the $\left\{f_{i}\right\}$. For the sake of simplicity, it is often assumed that the quality factors are equal for all the modes close to the frequency of analysis, approximating them with a composite quality factor [19]. This is, clearly, not physical, as the losses of each mode strongly depend on the field topography, a well-known fact in waveguide theory [17]; nevertheless, this approximation is usually capable of capturing the behaviour of a cavity and we will make use of a modified version in Appendix A. 


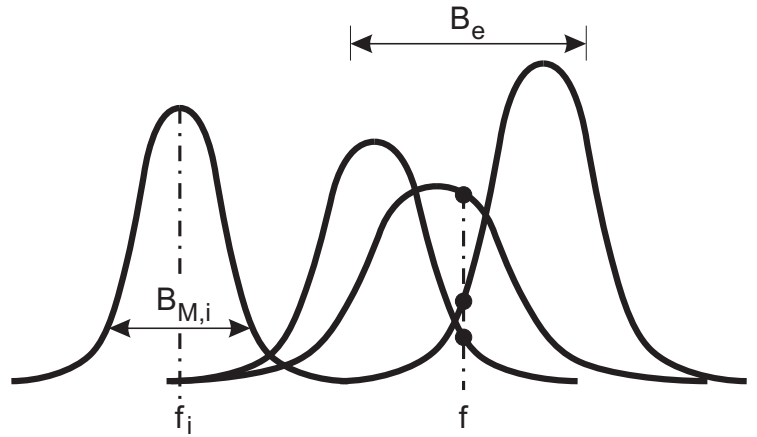

Fig. 1. A graphical depiction of the modal decomposition of a random realization of the electric field and some of the notations used. The dots represent the level of contribution of the three modes dominating the field at the working frequency $f$.

We will limit our analysis to a generic position $\mathbf{r}$. Introducing the following factorization for the modal topographies

$$
\mathbf{e}_{i}(\mathbf{r}, f)=e_{i}(\mathbf{r}, f) \hat{\boldsymbol{\xi}}_{i}(\mathbf{r}, f),
$$

we can hence restate (1) as

$$
\mathbf{E}(\mathbf{r}, f)=\sum_{i=1}^{\infty} \tilde{\gamma}_{i}(\mathbf{r}, f) \psi_{i}(f) \hat{\boldsymbol{\xi}}_{i}(\mathbf{r}, f),
$$

having introduced the equivalent weights

$$
\tilde{\gamma}_{i}(\mathbf{r}, f)=\gamma_{i}(f) e_{i}(\mathbf{r}, f)
$$

The second set of parameters is given by the modal weights $\left\{\tilde{\gamma}_{i}\right\}$, while the modal polarizations $\left\{\hat{\boldsymbol{\xi}}_{i}\right\}$ are the third and last set of parameters that we will consider.

The use of (4) allows studying field statistics in a simple way, as soon as the three modal parameter sets are treated as random variables. This approach is often used when studying the asymptotic properties of complex systems, as in [5], a common practice in statistical mechanics. Under this paradigm, (4) is entirely defined by the three sets of modal parameters $\left\{\tilde{\gamma}_{i}\right\}$, $\left\{f_{i}\right\},\left\{\hat{\boldsymbol{\xi}}_{i}\right\}$ and the composite quality factor $Q$.

Recalling that the contribution of each mode is weighted by its frequency response $\left\{\psi_{i}(f)\right\}$, the influence of each mode is localized around each resonance frequency $f_{i}$, as shown in Fig. 1. The extent of the influence of each mode is set by its quality factor $Q_{i}$, as the mode can be effectively excited only for working frequencies at most at a distance $\left|f_{i}-f\right|<$ $B_{M, i}$ from the resonance frequency. The distance $B_{M, i}$ is the bandwidth covered by each mode, from its peak at $f_{i}$, to a reduction of a factor $\rho$. Typical values of $\rho$ are $-3 \mathrm{~dB}$ and $-10 \mathrm{~dB}$. For the case of $\rho=-3 \mathrm{~dB}$,

$$
B_{M, i}=\frac{f_{i}}{Q_{i}},
$$

a result that will be used later.

As we will show, the introduction of the bandwidths $B_{M, i}$ is not necessary, nor the definition of a level $\rho$; nevertheless, this approach simplifies the mathematical derivation, while effectively pointing out that it is not necessary to carry out the sum in (4) over all of the modes, but just over a reduced subset $\mathscr{M}$

$$
\mathscr{M}=\left\{i:\left|\psi_{i}(f)\right|>\rho\left|\psi_{i}\left(f_{i}\right)\right|\right\} \quad,
$$

where $\rho$ is chosen in order to give a significant contribution from the modes. Hence, (4) is limited to a number of modes $M=\# \mathscr{M}$, i.e., the cardinality of $\mathscr{M}$, spanning a frequency bandwidth $B_{e}$

$$
B_{e}=\max _{i \in \mathscr{M}} B_{M, i},
$$

hereafter referred to as the equivalent bandwidth of the reverberation chamber. This concept will be shown to play an important role, as it accounts for the fact that a harmonic signal excites a number of modes that are to be found over this bandwidth. These concepts are illustrated in Fig. 1, for a random realization of (1).

\section{STATISTICAL MODAL ANALYSIS}

Following the previous discussions, we will consider the simplified model in (9) as the reference for our statistical analysis

$$
\mathbf{E}=\sum_{i \in \mathscr{M}} \tilde{\gamma}_{i} \psi_{i} \hat{\boldsymbol{\xi}}_{i}
$$

where the indexes $i$ now span the set $\mathscr{M}$, and having dropped the spatial and frequency dependencies, as our analysis will deal with the field statistics at one specific position and frequency at a time.

We focus our analysis on electric energy density

$$
W(\mathbf{r}, f)=\epsilon_{0}\|\mathbf{E}(\mathbf{r}, f)\|^{2},
$$

where $\epsilon_{0}$ is the dielectric constant for the medium filling the cavity, and the electric field is expressed in root-meansquare units. The model proposed in (9) is fit for studying any quantity related to the electric field. The rationale for choosing the electric energy density lies in its asymptotic convergence to a six-degree-of-freedom chi-square law, thus with a standardized variance equal to $1 / 3$, as opposed to the squared amplitude of Cartesian components (directly related to the received power for polarized electrically small antennas, such as dipoles) which follow a two-degree chi-square law, with a standardized variance equal to 1 . Although this has no impact from a theoretical point of view, it makes a big difference in practice, as the statistical uncertainty affecting moments estimated from a finite sample population is directly dependent on the relative statistical dispersion of the samples, as recalled in Appendix B. The experimental results presented in Section VI, dealing with the electric energy density, are indeed already affected by a non-negligible statistical uncertainty; use of single Cartesian components of the electric field would have resulted in a even higher uncertainty.

The average spatial uniformity properties of $W$ can be obtained straightforwardly from a continuous modal representation, as done in [5], where it is proven that $W$ follows a six-degree-of-freedom chi-squared probability law, or $\chi_{6}^{2}$. Although such approach allows to understand and explain in a simple way some of the most important properties of reverberation chambers, it is incapable of providing results but for asymptotic conditions. As such, it cannot explain why field statistics can deviate from the asymptotic $\chi_{6}^{2}$ probability law in practical scenarios, where the electromagnetic field within 
a reverberation chamber is given by a discrete plane-wave spectrum.

Let us now consider (9) under a similar statistical viewpoint. We will consider the three sets of modal parameters as random variables. The main assumptions required are that: 1) the modal parameters of different sets are independent and 2) the parameters within the same set are independent and identically distributed (iid). Recalling the physical meaning of these parameters, it is clear that they are not independent, as they are all related to the position and spatial distribution of the sources. Nevertheless this approach is commonly regarded as sound, and it is the foundation for statistical analysis for reverberation chambers [2].

The modal weights will be regarded as defined by an iid real and imaginary part

$$
\tilde{\gamma}_{i}=\alpha_{i}+\mathrm{j} \beta_{i}
$$

No specific assumption is required on the type of law followed by the $\left\{\alpha_{i}\right\}$ and $\left\{\beta_{i}\right\}$. We define

$$
\mu_{n}=\mathrm{E}\left[\left|\tilde{\gamma}_{i}\right|^{n}\right],
$$

as the $n$-th order moment of the modulus of the modal weights $\left\{\gamma_{i}\right\}$. These moments are identical for all the modal weights, following the iid assumption for the modal quantities.

The modal polarizations $\left\{\hat{\boldsymbol{\xi}}_{i}\right\}$ will be considered as uniformly distributed over a $4 \pi$-steradian angle, as done in [5], so that all polarizations are equally likely. Resonance frequencies will be assumed to be distributed uniformly over a bandwidth $B_{e}$ around the working frequency $f$. These are best-case assumptions, as they imply that a perfect stirring is available. Indeed, in order to meet these requirements, the stirring technique must be capable of providing perfectly uncorrelated samples, following exactly the same probability law. Hence, the following results are not only non-conservative, but rather optimistic, and they should be regarded as lower-bounds for any use in error estimation. Such choices are meant to model a perfect stirring technique, where for each random realization the frequencies of resonance of the cavity will be modified, with equal probability of finding them over the bandwidth $B_{e}$.

Actually, the probability density function for the $\left\{f_{i}\right\}$ should account for the fact that the probability of finding a resonance at a given frequency increases with the modal density of the cavity. As the modal density is not linear with frequency [2], resonance frequencies cannot, in general, be distributed uniformly. But as long as the bandwidth $B_{e}$ over which the $\left\{f_{i}\right\}$ are observed is small enough (highly-resonant cavity), the distribution can indeed be approximated as uniform. The actual problem with the use of simple probability density functions is that the phenomenon of mode clustering cannot be modelled properly. For the sake of simplicity this is going to be neglected in the remainder of this paper; again, this implies that we are setting our analysis in a best-case configuration, as mode clustering, would yield a stronger deviation from asymptotic results.

Modelling the $\left\{f_{i}\right\}$ as random variables leads to having $\left\{\psi_{i}(f)\right\}$ behaving as random functions. Subsequent analysis will show that their squared modulus play a central role.
Hence, we introduce the moments

$$
\nu_{n}=\mathrm{E}\left[\left|\psi_{i}(f)\right|^{n}\right],
$$

which, for $n=2$, represent the average power of the modes, and it accounts for how effectively they are made to resonate on average, as their frequencies of resonance $\left\{f_{i}\right\}$ are randomly scattered around the working frequency $f$. This should be regarded as a sort of available power, as the actual amount of power in the modes depends on the modal weights $\tilde{\gamma}_{i}$. At the same time, $\nu_{2}^{2}$ summarizes how power is shared among the different modes. Indeed the average mutual power shared by two any modes is

$$
\mathrm{E}\left[\left|\psi_{i}^{\star}(f) \psi_{j}(f)\right|^{2}\right]=\mathrm{E}\left[\left|\psi_{i}(f)\right|^{2}\right] \mathrm{E}\left[\left|\psi_{j}(f)\right|^{2}\right]=\nu_{2}^{2},
$$

recalling the independence assumption for the resonance frequencies $\left\{f_{i}\right\}$. As mutual power is a measure of the overlapping of the modes, it has an important place in field statistics.

Let us now consider (10). By introducing the Cartesian unit vectors $\hat{\boldsymbol{u}}_{k}$, the electric energy density $W$ can be written as

$$
W=\epsilon_{0} \sum_{k=1}^{3}\left|\mathbf{E} \cdot \hat{\boldsymbol{u}}_{k}\right|^{2} \text {. }
$$

Following (9)

$$
\begin{aligned}
W & =\epsilon_{0} \sum_{k=1}^{3}\left|\hat{\boldsymbol{u}}_{k} \cdot \sum_{i \in \mathscr{M}} \tilde{\gamma}_{i} \psi \hat{\boldsymbol{\xi}}_{i}\right|^{2} \\
& =\epsilon_{0} \sum_{k=1}^{3}\left\{\sum_{i \in \mathscr{M}}\left|\tilde{\gamma}_{i}\right|^{2}\left|\psi_{i}\right|^{2}\left|\hat{\boldsymbol{\xi}}_{i} \cdot \hat{\boldsymbol{u}}_{k}\right|^{2}+\right. \\
& \left.+\sum_{i \in \mathscr{M}} \sum_{j \in \mathscr{M}, j \neq i} \tilde{\gamma}_{i} \tilde{\gamma}_{j}^{\star} \psi_{i} \psi_{j}^{\star}\left(\hat{\boldsymbol{\xi}}_{i} \cdot \hat{\boldsymbol{u}}_{k}\right)\left(\hat{\boldsymbol{\xi}}_{j}^{\star} \cdot \hat{\boldsymbol{u}}_{k}\right)\right\}
\end{aligned}
$$

which can be restated as

$$
\begin{aligned}
W & =\epsilon_{0} \sum_{i \in \mathscr{M}}\left|\tilde{\gamma}_{i}\right|^{2}\left|\psi_{i}\right|^{2}+ \\
& +\epsilon_{0} \sum_{i \in \mathscr{M}} \sum_{j \in \mathscr{M}, j \neq i} \tilde{\gamma}_{i} \tilde{\gamma}_{j}^{\star} \psi_{i} \psi_{j}^{\star} \sum_{k=1}^{3}\left(\hat{\boldsymbol{\xi}}_{i} \cdot \hat{\boldsymbol{u}}_{k}\right)\left(\hat{\boldsymbol{\xi}}_{j}^{\star} \cdot \hat{\boldsymbol{u}}_{k}(\mathbf{1})\right.
\end{aligned}
$$

recalling that

$$
\sum_{k=1}^{3}\left|\hat{\boldsymbol{\xi}}_{i} \cdot \hat{\boldsymbol{u}}_{k}\right|^{2}=\left\|\hat{\boldsymbol{\xi}}_{i}\right\|^{2}=1
$$

This model can be used in order to study the actual role of losses in electric energy density statistics. To this end, we propose to compute the first two moments of the electric energy density, and to check under what conditions the standardized variance converges to the theoretical results recalled in [1], [2].

The average electric energy density can be computed by taking the ensemble average of (17). Recalling the assumption of independence between the modal weights and the frequencies of resonance, as well as the fact that the modal weights are iid random variables, we get

$$
\mathrm{E}[W]=\epsilon_{0} M \mu_{2} \nu_{2},
$$


having applied (12) and (13).

Getting on with the computation of the variance of the electric energy density, by squaring (17) and proceeding again by separating the coherent and incoherent parts of the sum, yields

$$
\begin{aligned}
\mathrm{E}\left[W^{2}\right] & =\epsilon_{0}^{2} M \mu_{4} \nu_{4}+ \\
& +\epsilon_{0}^{2} M(M-1) \mu_{2}^{2} \nu_{2}^{2}(1+ \\
& \left.+\mathbf{E}\left[\left|\sum_{k=1}^{3}\left(\hat{\boldsymbol{\xi}}_{i} \cdot \hat{\boldsymbol{u}}_{k}\right)\left(\hat{\boldsymbol{\xi}}_{j}^{\star} \cdot \hat{\boldsymbol{u}}_{k}\right)\right|^{2}\right]\right)
\end{aligned}
$$

The ensemble average in (20) can be simplified taking note of

$$
\sum_{k=1}^{3}\left(\hat{\boldsymbol{\xi}}_{i} \cdot \hat{\boldsymbol{u}}_{k}\right)\left(\hat{\boldsymbol{\xi}}_{j}^{\star} \cdot \hat{\boldsymbol{u}}_{k}\right)=\hat{\boldsymbol{\xi}}_{i} \cdot \hat{\boldsymbol{\xi}}_{j}^{\star}
$$

and since

$$
\mathrm{E}\left[\left|\hat{\boldsymbol{\xi}}_{i} \cdot \hat{\boldsymbol{\xi}}_{j}^{\star}\right|^{2}\right]=\frac{1}{3} \quad \forall i \neq j,
$$

equation (20) can be expressed as

$$
\mathrm{E}\left[W^{2}\right]=\epsilon_{0}^{2} M \mu_{4} \nu_{4}+\frac{4}{3} \epsilon_{0}^{2} M(M-1) \mu_{2}^{2} \nu_{2}^{2},
$$

so that the standardized variance is given by

$$
\left(\frac{\sigma}{\mu}\right)_{W}^{2}=\frac{\mathrm{E}\left[W^{2}\right]}{(\mathrm{E}[W])^{2}}-1=\frac{1}{M} \frac{\mu_{4}}{\mu_{2}^{2}} \frac{\nu_{4}}{\nu_{2}^{2}}+\frac{M-4}{3 M} .
$$

Based on the results demonstrated in Appendix A, the following result holds for $Q \gg 1$ (meaning several hundredths)

$$
\frac{\nu_{4}}{\nu_{2}^{2}} \simeq \frac{B_{e}}{\pi} \frac{Q}{f},
$$

where $Q$ is the composite quality factor of the cavity. Attention should be paid about the fact that the definition of this composite quality factor is not the same currently used in EMC, i.e., as defined in [8], [2]. In fact, this considers the average efficiency of a reverberation chamber in converting an input power into an electric energy density. This efficiency being based on the notion of average electric energy density over the test volume, it is not suitable for statistical convergence at a specific position. We rather deal with the average time constant of the cavity at a given point. Hence, it is a function of frequency and position, with a non-smooth behaviour in these variables, presenting a large dynamics of values. The notion of average is thus applied to the $Q_{i}$ of the dominant modes at the working frequency $f$.

From (25)

$$
\left(\frac{\sigma}{\mu}\right)_{W}^{2}=\frac{1}{\pi} \frac{\mu_{4}}{\mu_{2}^{2}} \frac{B_{e}}{M} \frac{Q}{f}+\frac{M-4}{3 M} .
$$

As we anticipated in the beginning of this Section, the definition of $B_{e}$ is redundant. As a matter of fact, (26) is approximated as long as we limit the sum in (9) to a finite number of modes around the working frequency. We should rather consider a level $\rho \rightarrow 0$, leading to $B_{e} \rightarrow \infty$ and, as a consequence, $M \rightarrow \infty$, i.e., let all the modes intervene. Clearly, this implies an increasing number of modes involved in (9), but with a level of energy getting lower as their frequency of resonance gets further away from the working frequency $f$. This is not in contradiction with our derivation, as this fact is accounted for by the $\nu_{n}$ moments. The use of the limit is valid as long as $Q$ is high enough to have the dominant modes confined into a narrow bandwidth around $f$, so that the idea of composite $Q$ is still physically acceptable.

Under these conditions, we can introduce the standardized variance $\varsigma_{W}^{2}$ of the electric energy density, taking the limit of (26) as

$$
\varsigma_{W}^{2}=\lim _{\rho \rightarrow 0}\left(\frac{\sigma}{\mu}\right)_{W}^{2}=\frac{1}{3}+\frac{1}{\pi} \frac{\mu_{4}}{\mu_{2}^{2}} \lim _{M \rightarrow \infty} \frac{B_{e}}{M} \frac{Q}{f} .
$$

Since for $Q \gg 1$

$$
\lim _{M \rightarrow \infty} \frac{B_{e}}{M}=\frac{1}{m(f)},
$$

where $m(f)$ is the modal density, expressed in $\mathrm{Hz}^{-1}$. Equation (27) can now be written as

$$
\varsigma_{W}^{2}=\frac{1}{3}+\frac{1}{\pi} \frac{\mu_{4}}{\mu_{2}^{2}} \frac{1}{M_{M}} .
$$

If a resonant mode were centered on $f$, thus with a $-3 \mathrm{~dB}$ bandwidth $B_{3 \mathrm{~dB}}=f / Q$, then $M_{M}=m(f) B_{3 \mathrm{~dB}}$ would be equal to the number of modes found on average within this bandwidth. In other words, $M_{M}$ assesses how strongly the modes overlap on average.

\section{ON THE OVERMODED CONDITION}

The result in (29) must be capable of predicting the asymptotic results expected from the theoretical and experimental analyses presented in [5], [20]. This is the case, as

$$
\lim _{M_{M} \rightarrow \infty} \varsigma_{W}^{2}=\frac{1}{3},
$$

which is the result expected for a $\chi_{6}^{2}$ probability distribution law [6]. Hereafter, we will refer to this asymptotic value as $\varsigma_{\chi_{6}^{2}}^{2}$.

This asymptotic result is met only when the number of overlapped modes increases, as opposed to common understanding, where modal density is regarded as the actual dominating parameter. This points to the true conditions that must be enacted for the cavity to be overmoded: requiring a large number of resonant modes is a necessary but not sufficient condition, as long as they are not overlapped. As this last event is tightly linked to the bandwidth of the mode response, the overmoded condition is strongly dependent on the losses experienced in the reverberation chamber.

The roles of the modal density and the composite quality factor $Q$ are clarified by Fig. 2, where the standardized variance of the electric energy density $\varsigma_{W}^{2}$ predicted by (29) is plotted against a varying modal density and quality factor. These results show how the idea of the overmoded region as a threshold condition could be easily thought as a correct definition, since increasing the modal density ultimately leads to an asymptotic convergence to the standardized variance expected for a $\chi_{6}^{2}$ law, i.e., the value $1 / 3$. 


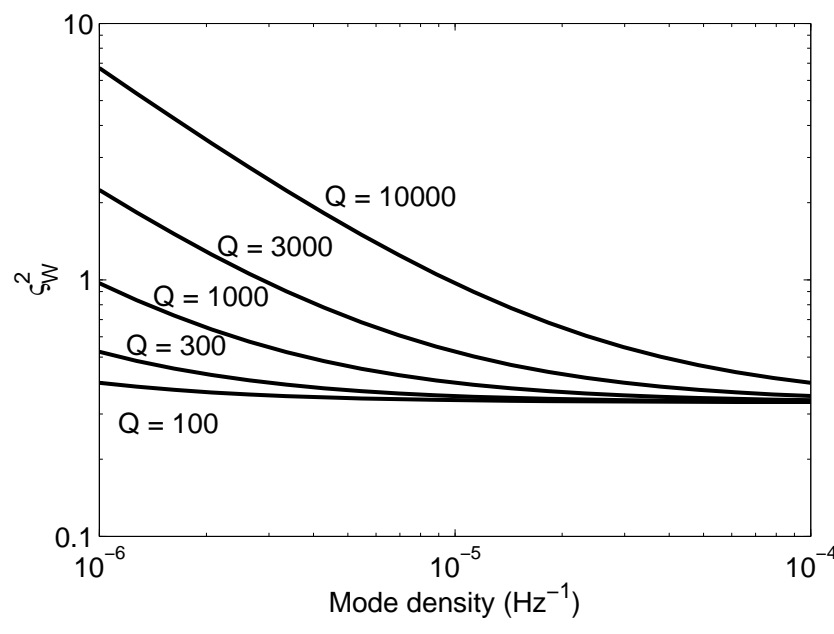

Fig. 2. The standardized variance predicted by (29), for a varying modal density and composite quality factor. A working frequency $f=1 \mathrm{GHz}$ was assumed.

It is noteworthy that common understanding looks at modal density and frequency as being univocally related: this wrong interpretation has likely originated because of the use of Weyl's formula [2], a smooth approximation not accounting for mode clusters. Actually, modal density broadly increases with frequency, but it can locally decrease or increase with respect to the smooth behaviour predicted by Weyl's formula, resulting in a non-monotonous function of frequency.

Fig. 2 also shows that an increasing modal density is not the only way of achieving the convergence in (30), since for a given modal density a relatively small increase of losses also leads to convergence.

This phenomenon is not new: in acoustics, this idea is expressed by Schroeder frequency [4], as the minimum frequency for which three modes are overlapped within their $-3 \mathrm{~dB}$ bandwidth. The problem is that this definition is arbitrary and unable to quantify how strongly the actual electric energy density statistics will diverge from the asymptotic results obtained for an infinite number of resonating modes.

As opposed to this approach, we consider the relative error $\epsilon_{\varsigma^{2}}$ between the result predicted by (29) and the asymptotic one

$$
\epsilon_{\varsigma^{2}}=\frac{\varsigma_{W}^{2}-\varsigma_{\chi_{6}^{2}}^{2}}{\varsigma_{\chi_{6}^{2}}^{2}}=\frac{3}{\pi} \frac{\mu_{4}}{\mu_{2}^{2}} \frac{1}{M_{M}} .
$$

The ratio $\mu_{4} / \mu_{2}^{2}$ is related to the kurtosis $\kappa$ of the real (or imaginary) part of the modal weights as

$$
\frac{\mu_{4}}{\mu_{2}^{2}}=\frac{1}{2}(\kappa+1) .
$$

In order to compute this error, we need to make some assumptions on the type of probability law followed by the modal weights. Although not justified by any physical phenomenon, they are usually assumed to be normally distributed [5]. Adopting this same approach $\mu_{4} / \mu_{2}^{2}=2$, yielding

$$
\epsilon_{\varsigma^{2}}=\frac{6}{\pi} \frac{1}{M_{M}}
$$

The ratio $\mu_{4} / \mu_{2}^{2}$ would not change much with the probability distribution law; e.g., for the case of uniformly distributed modal weights $\mu_{4} / \mu_{2}^{2}=7 / 5$.

The relationship between $M_{M}$ and the divergence from the asymptotic law is actually intuitive. Chi-squared laws are a direct consequence of the central-limit theorem, as recalled in [3], a condition approached as the number of degrees of freedom increases. For a cavity, modal representations provide a clear insight, as the number of degrees of freedom is just the number of modes effectively resonating (on average) at the working frequency. Clearly, this requires a potentially high number of modes (modal density), but also the possibility to make them resonate at the working frequency: this is directly dependent on the average quality factor of the modes, the dominant parameter for making a mode accessible when working at a frequency not equal to the one at which it resonates. The merit of (33) is that it provides a quantitative formula, indispensable in order to give a meaningful definition of the overmoded condition.

Indeed, (33) clearly shows that the overmoded condition is not given by a universal threshold, but rather dependent on the admissible error on the standardized variance. This topic will be the object of a more detailed discussion in Section V. If we consider a $10 \%$ error $\epsilon_{\varsigma^{2}}$ on the standardized variance as acceptable, then at least about 20 modes must overlap within their average $-3 \mathrm{~dB}$ bandwidth, centered around the working frequency. A quick computation allows to check that this condition if often not met in unloaded chambers, unless the working frequency is conspicuously higher than the (LUF). Experimental results supporting this claim are presented in Section VI.

These conclusions are coherent with the findings reported in [9], where it was shown that testing against the need of $\chi^{2}$ distribution laws, the minimum frequency for which the test is passed can be higher than the conventional LUF derived by applying the standard [8]. In a similar way, experimental results such as those presented in [15] go in this direction: they showed that by applying goodness-of-fit tests to the samples collected in what was considered as an overmoded cavity (in the sense of standard [8]), the test would fail for certain frequencies. This implied that the overmoded condition is not ensured by passing a threshold value, but that it depends more finely on the properties of the cavity at each frequency. Indeed, considering the paradigm we have introduced in this Section, the well-known fact that the composite quality factor of a cavity follows a frequency trend far from being smooth, implies that at frequencies where the quality factor increases, the probability of not passing a goodness-of-fit test can be expected to be higher. This conclusion is clearly submitted to the joint variation of the modal density and the composite quality factor, as the two can compensate each other.

The way (33) is defined implies that a high modal density can be a sufficient condition, when it goes to infinity, as required in asymptotic models [5]. But it also proves that for a given maximum error, the same statistical compliancy can be attained by controlling the losses within the cavity. As most of the time the modal density is not a design parameter, (33) can play an important role in the design of reverberation chambers. 
The attentive Reader should avoid thinking that (33) implies that increasing losses is a certain and good solution to the limitations of reverberation chambers. From an energetic point of view, increasing losses is obviously a non-desirable policy, as it would impair the ability of a reverberation chamber in efficiently generating a high-level electromagnetic field.

But at the same time, as demonstrated in Section VI, increasing losses provide some benefits, speeding up the convergence towards an asymptotic chi-square law. This notwithstanding, (33) holds true as long as modal-weight statistics and modal density can be regarded as unaffected by increased losses. Such condition is realistic if losses have a perturbative effect, implying a relatively small increase. Actually, this scenario occurs and is of interest in practice, as the inclusion of lossy EUTs within a reverberation chamber affects the statistics of the field the latter generates. The availability of a theory capable of predicting how the field statistics is modified should come of use in understanding under what conditions the behaviour of an unloaded chamber is not too sensitive to the inclusion of EUTs. This clearly is a matter of practical concern.

As opposed to the case of a perturbative effect, it has been highlighted how a strong increase in losses has a negative impact on field statistics, as in [21]. It is noteworthy that in that study the quality factor was reduced by a factor up to ten, thus strongly modifying the reverberation chamber behaviour, whose relationship with the unloaded configuration should be questioned. Again, our analysis is incapable of predicting how a strong reduction of the quality factor affects the modal description of a cavity, so that this type of effects are out of the scope of our work.

Attention should be paid to the fact that our analysis is optimistic, as it is based on the assumption that the stirrer technique be capable of ensuring that all the dominant modes will have the same probability to span the $-3 \mathrm{~dB}$ bandwidth around the working frequency. This means that in the case of mode clustering, the actual number of modes required might be higher.

\section{RELATIONSHIP WITH PROBABILITY-LAW TESTING}

The results presented so far assess the deviation from a $\chi_{6}^{2}$ law focusing on the standardized variance. Although this is a meaningful measure of statistical compliance, it is known from the "moment problem" that two probability laws can be expected to be identical only if all their moments are identical [22]. Hence, from a theoretical point of view, we cannot draw any conclusion on how close the empirical and asymptotic laws are, unless all of their moments were available. Our analysis is limited to the first two moments of the electric energy density; as the estimation of higher moments from experimental data is a critical issue [6], we regarded such approach as practically unfeasible, as the resulting higher moments would be overwhelmed by statistical uncertainty.

It is nevertheless fundamental to have a clue about how good (33) is as an estimator of the deviation of the entire probability law. As the actual (opposed to the asymptotic) probability distribution law of $W$ cannot be expressed in
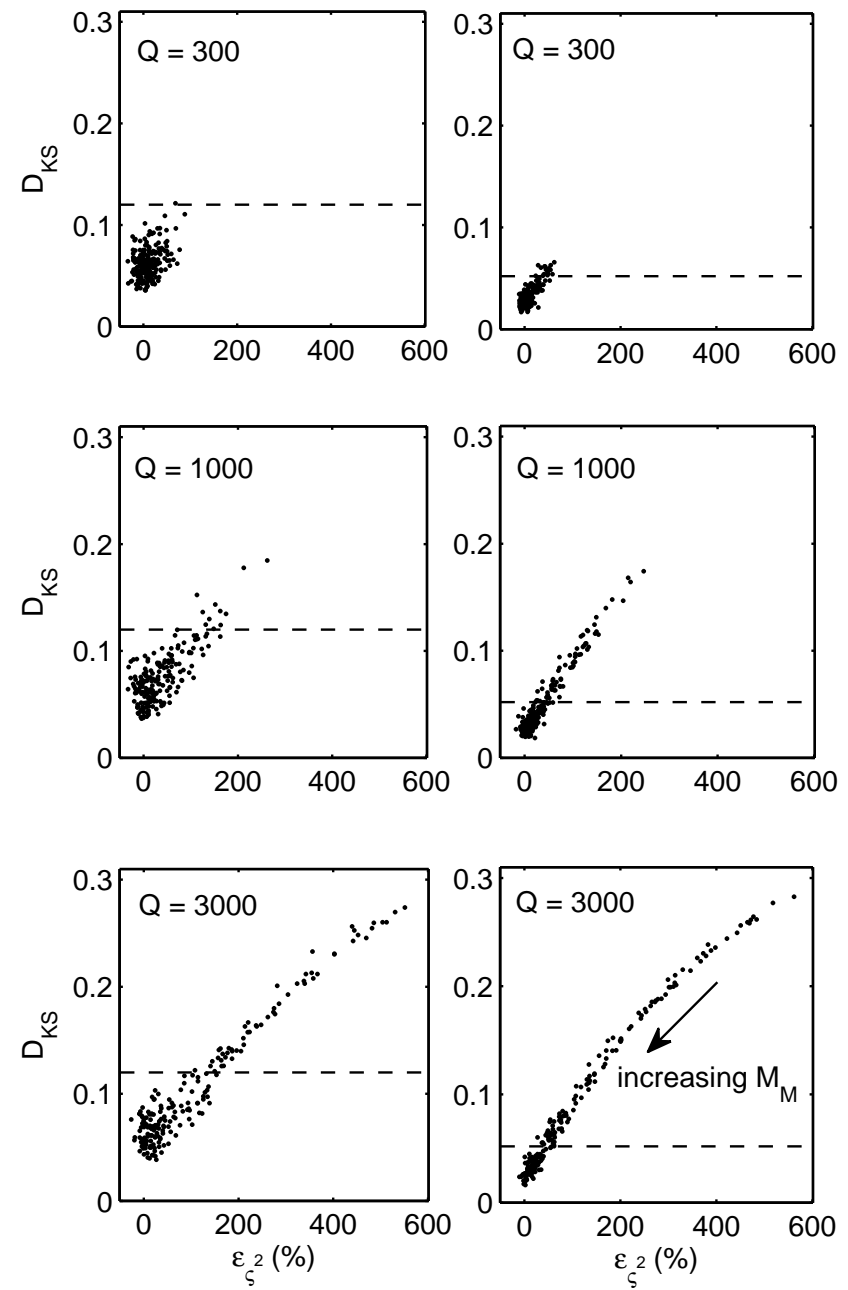

Fig. 3. Scatter plots representing the close relationship existing between the standardized variance of $W$ and Kolmogorov-Smirnov statistics $D_{K S}$ The model (9) was employed with three different values for the quality factor (300, 1000 and 3000), for 200 values of modal density, spanning the range from $10^{-6} \mathrm{~Hz}^{-1}$ to $10^{-4} \mathrm{~Hz}^{-1}$. For each configuration, 100 independent samples (left column) or 500 independent samples (right column) were generated, from which the relative error $\epsilon_{\varsigma^{2}}$ on the standardized variance and Kilmogorov-Smirnov statistics $D_{K S}$ were computed, and plotted as an individual point. All results have been computed for a working frequency $f=1 \mathrm{GHz}$. Dashed lines represent the threshold associated to KilmogorovSmirnov test, for accepting the null hypothesis of a $\chi_{6}^{2}$ distribution law with a $95 \%$ confidence margin.

closed-form, the link between these two quantities must be ascertained directly studying the electric energy density of field samples. To this end, we have used (9), generating random values for the three modal parameter sets (as introduced in Section III), obtaining a population of random samples for the electric energy density $W$, as generated within a perfectly stirred cavity. This allowed us to estimate two different pieces of information: 1) the standardized variance and 2) the standardized empirical distribution function $F_{W}(W)$ of $W$. From the latter we computed the Kolmogorov-Smirnov statistic $D_{K S}$, defined as [23]

$$
D_{K S}=\max _{W}\left|F_{W}(W)-F_{\chi_{6}^{2}}(W)\right| \quad,
$$




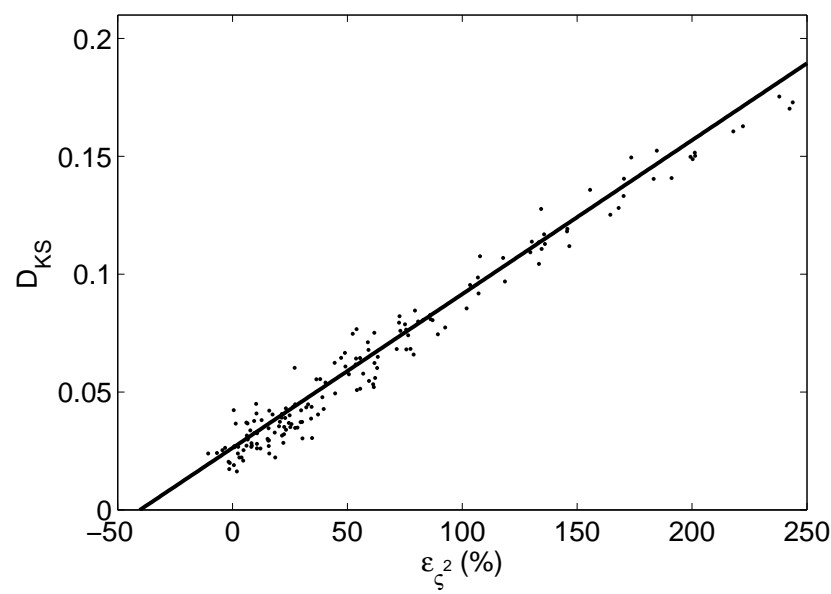

Fig. 4. Numerical data generated by (9), from 500-sample populations (as in Fig. 3), and the approximation (36) obtained by means of a least-squared linear regression (solid line).

where $F_{\chi_{6}^{2}}(W)$ is the standardized probability distribution function of the asymptotic $\chi_{6}^{2}$ law. Standardization of random variables, and hence of their associated probability distributions, is a necessary step, in order to apply goodness-of-fit tests in a meaningful way, as the reference asymptotic distribution moments are not known and configuration-dependent.

The correlation between $\epsilon_{\varsigma^{2}}$ and $D_{K S}$ was investigated by means of scatter plots, as those shown in Fig. 3. These results show unmistakably that the Kolmogorv-Smirnov statistics is tightly related to the error on the standardized variance. The parametric analysis in Fig. 3 proves that an increasing $Q$ leads to a stronger deviation from the asymptotic $\chi_{6}^{2}$ law. The scatter plots are actually parametric curves in the variable $M_{M}$, as pointed out in Fig. 3, rather than directly dependent on variables $m, f$ and $Q$. Hence, the results in Fig. 3 are not valid only for a specific configuration, but in general.

The fact that the points in Fig. 3 rather than laying on a curve are scattered should not be interpreted as a hint of a partial correlation between $D_{K S}$ and $\epsilon_{\varsigma^{2}}$ : as a matter of fact, these two quantities have been estimated from a finite population, implying that these estimators are affected by residual statistical uncertainty (see Appendix B). As a matter of fact, increasing the population from 100 to 500 samples shows a substantial reduction in the uncertainty of the data correlation. Hence, we think that the variance error $\epsilon_{\varsigma^{2}}$ could be used for assessing the deviation of the entire distribution law, although it only brings information about the first two moments of $W$. The accuracy of this approach is clearly dependent on the number of available samples.

In any case, the strong link between Kolmogorov-Smirnov statistics and the standardized variance error validates the idea of using (33) for predicting how changing losses would affect the statistical behaviour of a reverberation chamber, thus extending the purpose of (33) from an analysis tool for meaningful physical understanding, to potentially a prediction/design tool for practically ensuring the statistical compliance of a reverberation chamber.

The hypothesis of statistical compliance is based on the validity of the following condition [23]

$$
\sqrt{N} D_{K S}<K_{\alpha},
$$

where $N$ is the number of independent field samples and $K_{\alpha}$ is a threshold value for a significance level equal to $\alpha$. Typical values of $K_{\alpha}$ for $\alpha=0.05$ are about 1.15. This means that for $N \geqslant 100$, the most important region in Fig. 3 is for $\epsilon_{\varsigma^{2}} \lesssim$ $150 \%$, where the correlation between $\epsilon_{\varsigma^{2}}$ and $D_{K S}$ is close to linear. As establishing a closed-form expression linking these two quantities is likely difficult, we have rather opted for a simple linear regression model

$$
D_{K S} \simeq \eta_{1}+\eta_{2} \epsilon_{\varsigma^{2}} \quad \epsilon_{\varsigma^{2}} \geq 0,
$$

which is valid only for $\epsilon_{\varsigma^{2}} \lesssim 250 \%$. The regression parameters $\eta_{1}=2.6 \cdot 10^{-2}$ and $\eta_{2}=6.5 \cdot 10^{-2}$ refer to the model showed in Fig. 4.

Plugging (36) into (35) yields the maximum acceptable error $\epsilon_{\max }$ ensuring statistical compliance

$$
\epsilon_{\varsigma^{2}}<\epsilon_{\max } \frac{K_{\alpha}}{\eta_{2} \sqrt{N}}-\frac{\eta_{1}}{\eta_{2}} .
$$

Apart as a tool for checking the statistical compliance of a reverberation chamber, (37) is also important in the definition of the overmoded condition. As a matter of fact (37) states that in order to pass Kolmogorov-Smirnov test, it is not necessary to have a negligible error on the standardized variance. The actual upper-bound $\epsilon_{\max }$ to apply to $\epsilon_{\varsigma^{2}}$ can be quite high, as the number of samples $N$ decreases. This does not mean that the conclusions in Section IV are incorrect: as a matter of fact, this higher threshold just accounts for the fact that the true $\epsilon_{\varsigma^{2}}$ is not known, having been estimated from a finite population. Hence, it is pointless to try to enforce a condition on $\epsilon_{\varsigma^{2}}$ stronger than the precision with which this quantity is known.

As an example, using the data shown in Fig. 3, a 500-sample population would require a relative error $\epsilon_{\varsigma^{2}}<39 \%$ in order to accept the hypothesis of an electric energy density following the asymptotic chi-square distribution. Applying (33), this maximum error threshold is translated into a need for about 5 overlapped modes, a result well looser than the 20 modes required by setting a $10 \%$ error on the standardized variance. As a consequence, the definition of overmoded region cannot be dissociated from the statistical uncertainty that is inevitably present when dealing with estimators based on a finite population of random samples. In other words, it is not statistically meaningful to set a general and arbitrary threshold on the number of overlapped modes $M_{M}$.

Equation (37) can also be used for designing additional losses aiming at improving the statistics of the electric energy density. Given a working frequency $f$ and an estimate of the modal density $m$, and computing from (37) the maximum error $\epsilon_{\max }$ leading to passing the Kolmogorov-Smirnov test, a maximum composite quality factor $Q_{\max }$ is found

$$
Q_{\max }=\frac{\pi}{6} m f \epsilon_{\max } .
$$

Considering a lower $Q$ would be pointless, as the improvement on statistics would be undetectable, due to unavoidable statistical uncertainty, while field-strength would degrade. 


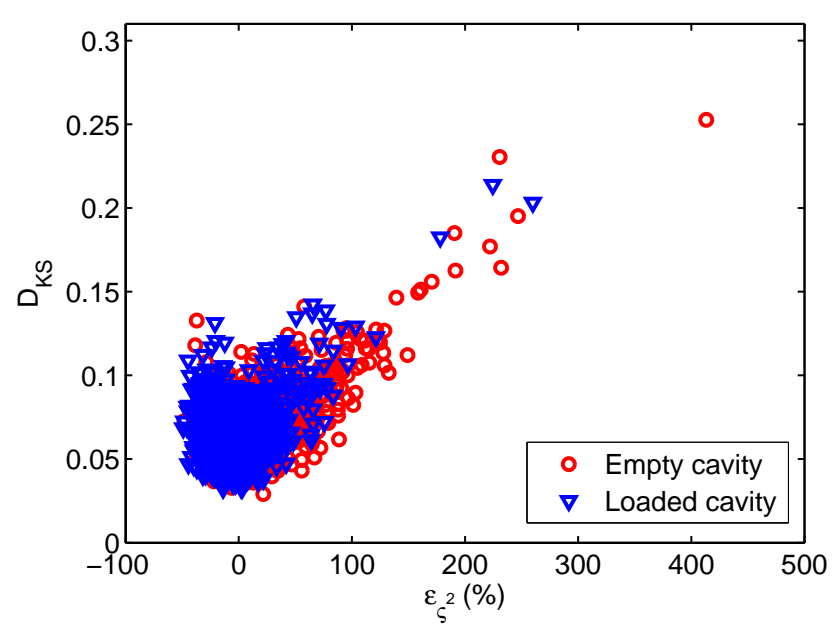

Fig. 5. Scatter plots of $D_{K S}$ and $\epsilon_{\varsigma^{2}}$ as derived from experimental data for the case of the empty and absorber-loaded chamber. The samples are related to the entire frequency range $0.7-3 \mathrm{GHz}$.

These discussions clearly hold as long as (9) is physically sound, i.e., as additional losses have a perturbative effect on the field within the cavity. An experimental validation of this model is presented in the next Section.

\section{EXPERIMENTAL RESULTS}

In order to validate our findings, experimental tests were carried out in Supelec's reverberation chamber. This cavity, measuring $13.3 \mathrm{~m}^{3}$, and equipped with a 100-step mechanical stirrer, has its LUF around $550 \mathrm{MHz}$. In our setup, a $\log$ periodic dipole antenna was used as a source exciting the cavity, over the frequency range $0.7-3 \mathrm{GHz}$. An optical-link field probe was used in order to collect data about the three field components at one position within the test volume of the chamber, while the stirrer was made to move over its entire range of rotation.

This approach was used for two configurations, for an empty cavity and with a small piece of RF absorber, made up of 4 pyramids about $30 \mathrm{~cm}$ high, standing in the center of the floor of the cavity. As the field probe used was phase sensitive, we were able to compute the composite quality factor for the cavity over the entire frequency range of test, by postprocessing the frequency-spectrum data in time domain (see Fig. 6).

The field samples were used in order to compute the electric energy density samples. The same procedure exposed in the previous Section was then applied: the aim was to check whether the same correlation between $D_{K S}$ and $\epsilon_{\varsigma^{2}}$ was to be found in practice. The results of this analysis, shown in Fig. 5, confirm those presented in Fig. 3. As the number of steps is limited to 100 , the statistical uncertainty associated to the estimations of $\epsilon_{\varsigma^{2}}$ and $D_{K S}$ is not negligible, as already discussed in the previous Section and detailed in Appendix B. Moreover, the actual number of independent samples generated by the mechanical stirrer is frequencydependent, going from about 30 around $700 \mathrm{MHz}$, to about 100

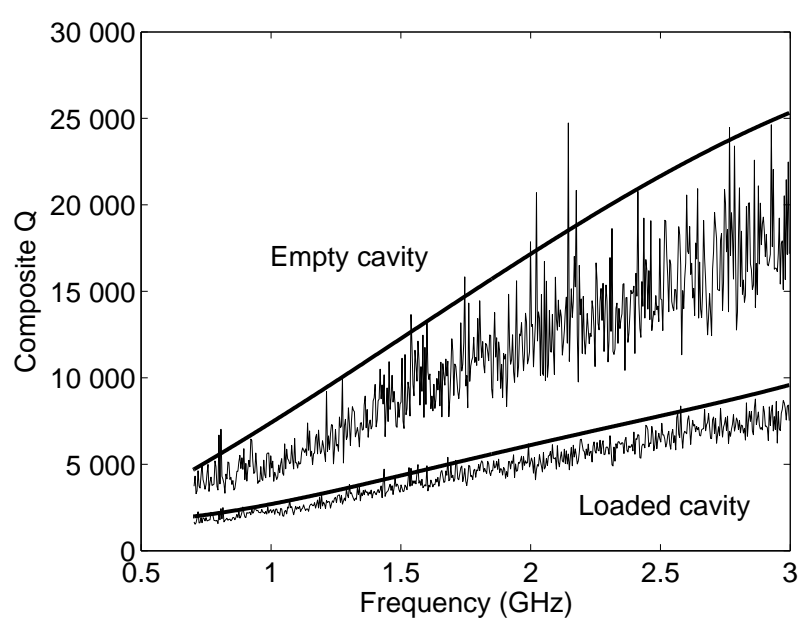

Fig. 6. Quality factors for the empty and loaded chambers. The smooth curves represent the loose majorants used for deriving the maximum-error results in Fig. 7.

at $3 \mathrm{GHz}$, thus leading to an even higher statistical uncertainty in the lower frequency range.

Having validated the close relationship between $D_{K S}$ and $\epsilon_{\varsigma^{2}}$, we went further in our validation by focusing on the relative error $\epsilon_{\varsigma^{2}}$. The next step was to look at how well (33) allows to predict the maximum deviation of the standardized variance, knowing a fair estimate of the modal density and the composite quality factor of a reverberation chamber. Though the latter can be estimated by means of measurements, modal density is not something that is routinely measured, although a solution to this problem has recently been proposed [24] In the context of this paper, we have stuck to the current approach consisting in using Weyl's approximation, and we have considered the simplest of Weyl's formulas [2]

$$
m(f) \simeq \frac{8 \pi V f^{2}}{c^{3}}
$$

With no access to a precise estimate of modal density, any attempt at finely predicting the standardized deviation error is bound to an error that cannot be estimated easily. For this reason we rather focused on the ability to provide results bounding the error, and thus capable of giving a warning about the global trend of $\epsilon_{\varsigma^{2}}$ over a given frequency range. Following this point of view, rather than using the quality factor estimate obtained from experimental characterization of our chamber, we considered a smooth majoring curve. The rationale behind this approach is that, according to (33), the maximum error occurs when a minimum number of overlapped modes is present, which in turn occurs when the ratio of the modal density and the composite quality factor is at a minimum. The use of a smooth curve is justified by our interest in the trend of the error, and not its fine modelling. Furthermore, the information available on the quality factor is often provided by simple predictive models [2], particularly during a design phase.

Fig. 6 shows that we did not use a true majorant. The reason for this is that it would have provided too conservative results, as a few points higher than average can lead to a 


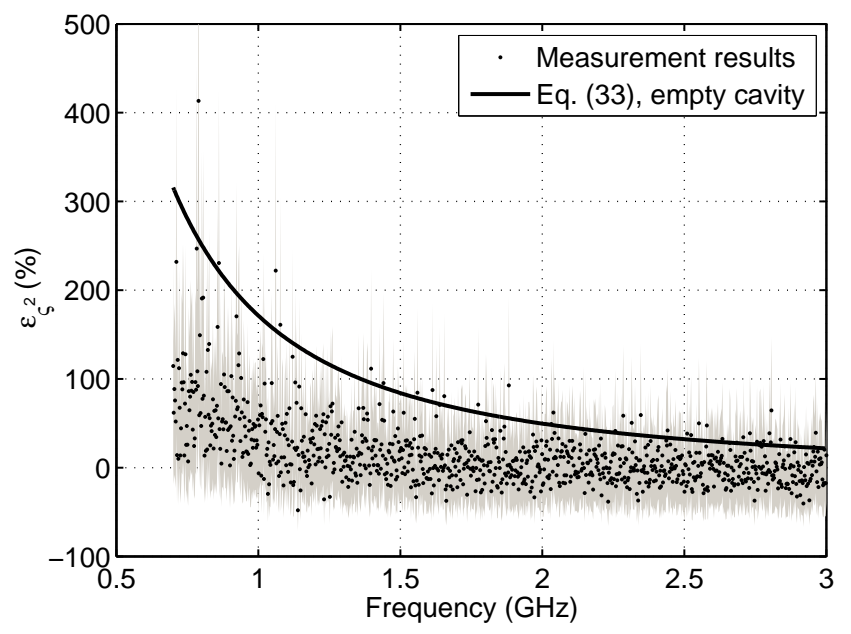

(a)

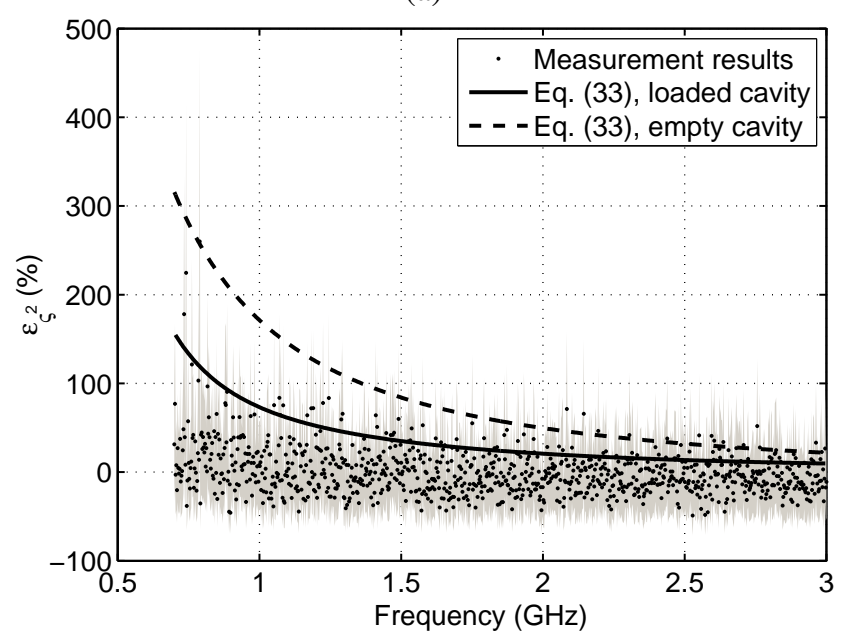

(b)

Fig. 7. Experimentally estimated relative error $\epsilon_{\varsigma^{2}}$ as a function of frequency: (a) for the empty cavity and (b) for the one loaded with the small absorber The results from (33) have been computed from the smooth curves majoring the composite Q derived from the experimental data and shown in Fig. 6. Shaded areas stand for the $95 \%$ statistical uncertainty of the estimated $\epsilon_{\varsigma^{2}}$, computed for a $95 \%$ confidence margin as shown in Appendix B.

strong overestimation of $Q$. The approximations we employed were chosen as a compromise between the need of a majoring curve and that of not considering a too strongly overestimated quality factor. As a consequence, for certain frequencies the relative error $\epsilon_{\varsigma^{2}}$ can be higher than the estimated upper bound. This outcome can also be caused by modal depletion, whose frequencies of occurrence are unknown.

From these data and (39) we computed the curves shown in Fig. 7, predicting the maximum deviation of the electric energy density from the asymptotic chi-square law. The actual error $\epsilon_{\varsigma^{2}}$ was directly estimated from the experimental data. The statistical uncertainty associated to these results has been estimated with a $95 \%$ confidence margin as detailed in Appendix B, and is shown in Fig. 7 as shaded areas.

Fig. 7 proves that (33) is indeed capable of predicting the trend of the maximum $\epsilon_{\varsigma^{2}}$, as the composite quality factor and the modal density evolve in frequency, and this for two

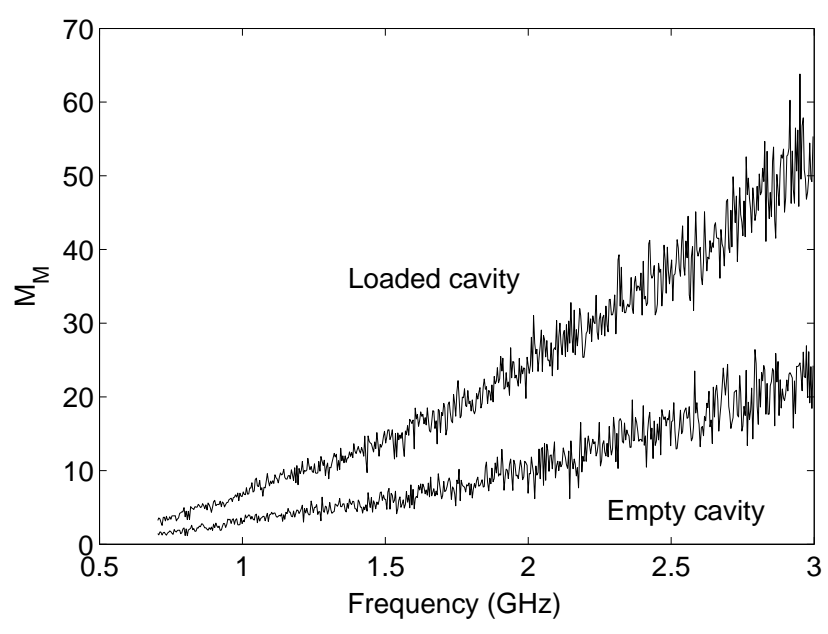

Fig. 8. Estimated average number of overlapped modes $M_{M}$, for the unloaded and loaded configurations.

chamber configurations. The comparison between the error $\epsilon_{\varsigma^{2}}$ in the case of the empty and loaded chamber is especially interesting as it is clear that the results predicted by (33) are indeed closely following the global trend of the maximum deviation of the statistics of the electric energy density. These results also prove that (33) can be used in practice as a design tool, as the majorant of the error computed in the case of inclusion of additional losses correctly predicts the improvement in the worst-case statistical performance of the loaded cavity.

follow a $\chi_{6}^{2}$ distribution law, a residual statistical uncertainty would be present.

The statistical uncertainty cannot be neglected, as it is the main reason for the residual error at the higher frequency range. The procedure proposed in Appendix B allows estimating a residual error of about $46 \%$ on $\epsilon_{\varsigma^{2}}$, even though this latter is expected to be close to zero. This result is independent of the proposed model, and merely based on statistical considerations. This statistical uncertainty, present even when then reverberation chamber is expected to behave ideally, is the reason of existence of the ambiguity in the definition of the overmoded condition. It is meaningless to require an error $\epsilon_{\varsigma^{2}}$ smaller than this statistical uncertainty, as the former cannot be measured precisely enough. It is interesting to notice that this statistical uncertainty appears to be smaller than the threshold imposed by (37) for accepting the asymptotic condition (and thus the overmoded condition), as it would be expected, since they are intimately related.

Looking at the $\epsilon_{\varsigma^{2}}$ estimated from measurements, there exist a lower frequency for which the error seems not to reduce anymore. This frequency is about $2 \mathrm{GHz}$ and $1.5 \mathrm{GHz}$ for, respectively, the empty and loaded chambers. It is worthwhile checking what is the number of overlapped modes $M_{M}$ at these frequencies. An estimate of $M_{M}$ is shown in Fig. 8, based on the experimentally evaluated composite quality factors and the modal density given by (39). For the two frequencies previously mentioned, it seems that a minimum number of 10 overlapped modes is required, in order to have the 
better performance possible, according to the limited accuracy provided by the residual statistical uncertainty. These results are actually too restrictive, as a more statistically motivated choice would make use of (37), obtaining $800 \mathrm{MHz}$ for the empty chamber and a frequency below $700 \mathrm{MHz}$ for the loaded one. This example is meaningful in depicting the intrinsical ambiguity of a single definition of the overmoded condition, and the fundamental insight brought in by (33) and (37).

A final discussion is worthwhile: the validity of (33) implies that if the error it predicts is comparable with the one found in practice in a mode-stirred reverberation chamber, than it would be wrong, from a statistical point of view, to conclude anything about the eventual inefficiency of the stirring technique. As a matter of fact, (33) has been derived under a perfect-stirring assumption, so that the eventual presence of a poor stirring is expected to provide an error $\epsilon_{\varsigma^{2}}$ higher than that due to a limited modal overlapping. This is all the more true in the lower frequency range, where the ineffectiveness of field stirring is often regarded as the major source of statistical noncompliancy in reverberation chambers: interestingly, the lower frequency range is also where poor modal overlapping appears more strongly.

\section{CONCLUSIONS}

A discrete modal description of the field within a cavity has allowed us to quantify the role that losses play in the statistics of the electric energy density generated within reverberation chambers. The proposed model has led to the derivation of a simple formula expressing the error between the actual standardized variance and the asymptotic one. This error was shown to be dominated by the number of modes superposed within the $-3 \mathrm{~dB}$ bandwidth of the dominant modes; as such, this result goes against common understanding that the overmoded condition is a mere matter of available resonating modes. We have proven that the definition of the overmoded condition is not universal, but depends on the maximum acceptable deviation from asymptotic laws, as well as on the number of independent samples generated by the stirring technique.

By linking the standardized variance error $\epsilon_{\varsigma^{2}}$ to Kolmogorov-Smirnov statistics, it was proven that $\epsilon_{\varsigma^{2}}$ is a meaningful metric for assessing how likely electric energy density samples are to deviate from an asymptotic $\chi_{6}^{2}$ probability law. This same analysis has led to the definition of a maximum composite quality factor that should not the exceeded in order to ensure statistical compliancy. A fundamental result is that even starting with a perfect-stirring assumption and a high modal density, an electrically large reverberation chamber can still present a non-asymptotic statistical performance. These conclusions have major consequences on other commonly accepted ideas, such as that non-compliance with asymptotic laws is always a matter of poor stirring and that the $Q$ should always be as high as possible.

Experimental results support our findings, both for the soundness of the proposed deviation metric and the fact that it allows to predict the actual error incurred in field samples. This latter result is fundamental, as it could lead to a simple way of assessing the statistical compliance of a reverberation chamber, and how relatively small changes in its composite quality factor would affect its statistics.

\section{APPENDIX A}

MOMENTS OF THE $\left|\psi_{i}(f)\right|$ RANDOM VARIABLES

Following the derivation given in Section IV, two moments are needed, the second and the fourth. The second-order moment of $\left|\psi_{i}(f)\right|$ reads

$$
\mathrm{E}\left[\left|\psi_{i}(f)\right|^{2}\right]=\int\left|\psi_{i}(f)\right|^{2} p\left(f_{i}\right) \mathrm{d} f_{i},
$$

where $p\left(f_{i}\right)$ is the probability density function for the frequency of resonance $f_{i}$. As recalled in [11], there exist minimum requirements for the composite quality factor of a cavity, for it to be compliant with EMC standards. This being coherent with our study, we can assume $Q_{i} \gg 1$. As a consequence, the average relative bandwidth $B_{M, i}=f_{i} / Q_{i}$ of each mode can be expected to be much smaller than one. Hence, the $\left|\psi_{i}(f)\right|$ give a non-negligible contribution over an equivalent bandwidth $B_{e} / f_{i}=1 / Q_{i} \ll 1$. The ensemble integral in (40) can thus be limited over a finite and narrow bandwidth $B_{e}$; this implies that it is reasonable to assume that over this bandwidth all of the modes have the same bandwidth In other words, one can consider that the $\psi_{i}(f)$ functions are frequency-shifted replicas of the same template $\psi_{0}(f)$, i.e., $\psi_{i}(f)=\psi_{0}\left(f-f_{i}\right)$. At the same time, the narrow-band requirement, together with the perfect-stirrer assumption at the heart of our work, allows to consider resonance frequencies uniformly distributed over $B_{e}$. As such

$$
\mathrm{E}\left[\left|\psi_{i}(f)\right|^{2}\right]=\frac{1}{B_{e}} \int_{B_{e}}\left|\psi_{i}(f)\right|^{2} \mathrm{~d} f_{i} .
$$

The frequency-replica paradigm implies that

$\int_{B_{e}}\left|\psi_{i}(f)\right|^{2} \mathrm{~d} f_{i}=\int_{B_{e}}\left|\psi_{0}\left(f-f_{i}\right)\right|^{2} \mathrm{~d} f_{i}=\int_{B_{e}}\left|\psi_{0}(f)\right|^{2} \mathrm{~d} f$.

The last integral is now recognizable as the energy of the template function; clearly, this leads to modal functions $\psi_{i}(f)$ with the same energy. This was shown to be given by [25]

$$
\int_{B_{e}}\left|\psi_{0}(f)\right|^{2} \mathrm{~d} f=\frac{1}{B_{M, i}} \frac{\pi}{2} \frac{Q_{i}^{2}}{Q_{i}^{2}+1} \simeq \frac{\pi}{2 B_{M, i}} .
$$

Hence

$$
\nu_{2} \simeq \frac{\pi}{2 B_{M, i} B_{e}}
$$

The fourth-order moment can be obtained by means of the same approach [25], yielding

$$
\nu_{4} \simeq \frac{\pi}{4 B_{M, i}^{3} B_{e}}
$$

and finally

$$
\frac{\nu_{4}}{\nu_{2}^{2}} \simeq \frac{B_{e}}{\pi} \frac{Q}{f}
$$

having introduced the approximation $B_{M, i} \simeq B_{M}$, where $B_{M}=f / Q$ is the average $-3 \mathrm{~dB}$ bandwidth of the dominant modes at the working frequency $f$ and $Q$ the associated composite quality factor. 


\section{APPENDIX B}

\section{STATISTICAL UNCERTAINTY FOR A FINITE POPULATION}

In the experimental evaluation of the error $\epsilon_{\varsigma^{2}}$ in the standardized variance, estimators are used for the average value and the variance of $W$. Having been derived from a finite population of $N$ independent samples, these estimators are affected by a residual statistical uncertainty that is important to acknowledge and estimate. This can be done by applying a local linearization of the definition of the error $\epsilon_{\varsigma^{2}}$, obtaining the following propagation-of-error model [6]

$$
\sigma_{\hat{\epsilon}_{\varsigma^{2}}}^{2}=\left(\frac{\partial \epsilon_{\varsigma^{2}}}{\partial \mu_{W}}\right)^{2} \sigma_{\hat{\mu}_{W}}^{2}+\left(\frac{\partial \epsilon_{\varsigma^{2}}}{\partial \sigma_{W}^{2}}\right)^{2} \sigma_{\hat{\sigma}_{W}^{2}}^{2},
$$

where $\hat{\mu}_{W}$ and $\hat{\sigma}_{W}^{2}$ are unbiased estimators of, respectively, $\mu_{W}$, the average electric energy density and $\sigma_{W}^{2}$, its variance, as derived from the $N$ available samples [6]. The derivatives are evaluated over the average values of these estimators. These estimators behave as random variables, with average values equal to those they should estimate (unbiased estimators) and variances $\sigma_{\hat{\mu}_{W}}^{2}$ and $\sigma_{\hat{\sigma}_{W}}^{2}$. Assuming the $N$ samples to be iid, the estimator variances can be approximated as [6]

$$
\begin{aligned}
\sigma_{\hat{\sigma}_{W}^{2}}^{2} & =\sigma_{W}^{4}\left(\frac{2}{N-1}+\frac{\kappa_{W}-3}{N}\right) \\
\sigma_{\hat{\mu}_{W}}^{2} & =\frac{\sigma_{W}^{2}}{N}
\end{aligned}
$$

where $\kappa_{W}$ is the kurtosis of the random variable $W$. As this value is not known for the random variable $W$, we will approximate it by means of its asymptotic value for a $\chi_{6}^{2}$ distribution law, i.e., $\kappa_{W}=5$. This yields

$$
\sigma_{\hat{\epsilon}_{\varsigma^{2}}}^{2} \simeq \frac{4}{3 N}\left(1+\epsilon_{\varsigma^{2}}\right)^{3}+\left(\frac{2}{N-1}+\frac{\kappa_{W}-3}{N}\right)\left(1+\epsilon_{\varsigma^{2}}\right)^{2},
$$

which is the square of the root-mean-square uncertainty of the $\epsilon_{\varsigma^{2}}$ estimator. Attention should be paid to the fact that (50) depends on the exact error $\epsilon_{\varsigma^{2}}$, which is only known by means of its estimate $\hat{\epsilon}_{\varsigma^{2}}$. In the higher frequency range, as $\epsilon_{\varsigma^{2}} \rightarrow 0$, (50) simplifies to

$$
\lim _{\epsilon_{\varsigma^{2}} \rightarrow 0} \sigma_{\hat{\epsilon}_{\varsigma^{2}}}^{2} \simeq \frac{16}{3 N}
$$

which is the residual statistical uncertainty that hinders the enforcement of a single definition of the overmoded condition. For $N=100$ the standard deviation of the estimation error is about $23 \%$. Assuming it to be normally distributed, the maximum residual error is about twice as such, for a $95 \%$ confidence level, i.e., about $46 \%$.

\section{REFERENCES}

[1] J. Kostas and B. Boverie, "Statistical model for a mode-stirred chamber," Electromagnetic Compatibility, IEEE Transactions on, vol. 33, no. 4, pp. $366-370$, nov 1991

[2] D. Hill, "Electromagnetic theory of reverberation chambers," NIST Technical note, no. 1506, 1998.

[3] T. Lehman, "A statistical theory of electromagnetic fields in complex cavities," Interaction Notes, Note 494, 1993.

[4] M. Schroeder, "Statistical parameters of the frequency response curves of large rooms," J. Audio Eng. Soc, vol. 35, no. 5, pp. 299-305, 1987.
[5] D. Hill, "Plane wave integral representation for fields in reverberation chambers," IEEE Transactions on Electromagnetic Compatibility, vol. 40, no. 3, pp. 209-217, 1998.

[6] F. James, Statistical methods in experimental physics. World Scientific, 2006.

[7] N. Wellander, O. Lundén, and M. Bäckström, "Experimental investigation and mathematical modeling of design parameters for efficient stirrers in mode-stirred reverberation chambers," Electromagnetic Compatibility, IEEE Transactions on, vol. 49, no. 1, pp. 94 -103, feb. 2007.

[8] Reverberation chamber test methods, International Electrotechnical Commission (IEC) Std. 61 000-4-21, 2003.

[9] C. Lemoine, P. Besnier, and M. Drissi, "Investigation of reverberation chamber measurements through high-power goodness-of-fit tests," Electromagnetic Compatibility, IEEE Transactions on, vol. 49, no. 4, pp. 745 -755 , nov. 2007

[10] G. Orjubin, E. Richalot, S. Mengue, and O. Picon, "Statistical model of an undermoded reverberation chamber," Electromagnetic Compatibility, IEEE Transactions on, vol. 48, no. 1, pp. 248 -251, feb. 2006.

[11] C. Holloway, D. Hill, J. Ladbury, and G. Koepke, "Requirements for an effective reverberation chamber: unloaded or loaded," Electromagnetic Compatibility, IEEE Transactions on, vol. 48, no. 1, pp. $187-194$, feb. 2006.

[12] B. Zhang, W. Li, X. Li, Z. Yuan, J. He, and R. Zeng, "Load effect investigation of a reverberation chamber," in Antennas, Propagation and EM Theory, 2008. ISAPE 2008. 8th International Symposium on, November 2008, pp. 1115-1118.

[13] D. Zhang, E. Li, T. Yeo, W. Chow, and J. Quek, "Influences of loading absorber on the performances of a reverberation chamber," in Electromagnetic Compatibility, 2003 IEEE International Symposium on, vol. 1, August 2003, pp. 279-281 vol.1.

[14] O. Lundén and M. Bäckström, "How to avoid unstirred high frequency components in mode stirred reverberation chambers," in Electromagnetic Compatibility, 2007. EMC 2007. IEEE International Symposium on, July 2007, pp. 1-4.

[15] V. Primiani, F. Moglie, and V. Paolella, "Numerical and experimental investigation of unstirred frequencies in reverberation chambers," in Electromagnetic Compatibility, 2009. EMC 2009. IEEE International Symposium on, August 2009, pp. 177-181.

[16] J. Dawson, T. Konefal, M. Robinson, A. Marvin, S. Porter, and L. Chirwa, "Field statistics in an enclosure with an aperture - effect of Q-factor and number of modes," in Electromagnetic Compatibility, 2005. EMC 2005. 2005 International Symposium on, vol. 1, 8-12 2005, pp. $141-146$ Vol. 1.

[17] N. Marcuvitz, Waveguide handbook. Inspec/Iee, 1986.

[18] J. Van Bladel, Electromagnetic fields. IEEE, 2007.

[19] B. Liu, D. Chang, and M. Ma, "Eigenmodes and the composite quality factor of a reverberating chamber," US Nat. Bur. Stand. Tech. Note, vol. 1066, 1983.

[20] P. Corona, G. Ferrara, and M. Migliaccio, "Reverberating chambers as sources of stochastic electromagnetic fields," Electromagnetic Compatibility, IEEE Transactions on, vol. 38, no. 3, pp. 348 -356, Aug 1996.

[21] O. Lundén and M. Bäckström, "Absorber loading study in FOI $36.7 \mathrm{~m}^{3}$ mode stirred reverberation chamber for pulsed power measurements," in IEEE EMC Symposium, Detroit, USA, 18-22 August 2008.

[22] K. Chung, A course in probability theory. Academic Pr, 2001.

[23] R. D'Agostino and M. Stephens, Goodness-of-fit techniques. CRC, 1986.

[24] A. Cozza, "An experimental method for assessing the modal density in a reverberation chamber," in Electromagnetic Compatibility (APEMC), 2010 Asia-Pacific Symposium on, 12-16 April 2010, pp. 578 -581.

[25] _ - "Statistics of the performance of time reversal in a lossy reverberating medium," Physical Review E, vol. 80, no. 5, p. 56604, 2009. 